\title{
A Fast and Accurate Workflow for Analytic 3D FIB-SEM Tomography
}

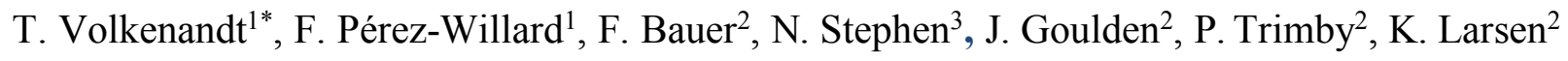 \\ 1. Carl Zeiss Microscopy GmbH, Oberkochen, Germany. \\ 2. Oxford Instruments NanoAnalysis, High Wycombe, UK. \\ 3. University of Plymouth, Plymouth, UK. \\ * Corresponding author: tobias.volkenandt@zeiss.com
}

The integration of energy dispersive spectrometry (EDS) and electron backscattered diffraction (EBSD) on a scanning electron microscope (SEM) are routine methods of material analysis. EDS offers chemical quantification and element spatial distribution in the form of quantitative analysis and X-ray mapping. EBSD enables microstructural characterization studying phase relationships, local mis-orientations and grain properties such as size, morphology and boundary characteristics. The integration of these two techniques on a single platform with simultaneous acquisition enables full material characterization and data correlation within a single user interface.

Development in automating these analysis techniques with focused ion beam (FIB) technology has extended this powerful material characterization into the third dimension. This is invaluable in applications where a true 3D understanding of the sample is required [1]. This analysis follows an iterative process, whereby the ion beam removes a slice of material before an electron image, EDS and EBSD map are acquired, a schematic of this process is shown in Figure 1. This workflow generates a slice by slice stack of data that can be processed post acquisition to reconstruct a representation of the volume of interest in $3 \mathrm{D}$.

The software controlling the acquisition of the analytic tomography offers a unique system to track and adjust the slice thickness in real time. To achieve this, a pair of angled fiducial lines is patterned in advance and imaged during the run. The measurement of the distance between these lines allows to calculate the thickness of the milled slice and to estimate the current sample drift. The milling progress is then adjusted accordingly to assure homogeneous slice thicknesses and compensate for sample drift. This system provides optimal resolution in $\mathrm{Z}$ direction. To achieve optimal resolution also in $\mathrm{XY}$ for the imaging, it is necessary to reduce the primary beam energy and make use of advanced detectors capable of filtering the detected electrons. These conditions, however, are not ideal for high signal-to noise EBSD map acquisition. Therefore, a new feature has been introduced that allows setting a second set of conditions for the EBSD map acquisition. These conditions typically include higher primary beam energies and larger beam currents. The switching between the two sets of conditions happens automatically during the run, while advanced algorithms assure perfect repositioning and fine tuning of the beam. This way, SEM images and EBSD maps are acquired under their ideal conditions respectively. The development of next generation CMOS based EBSD detectors and large area EDS detectors which can collect high quality data at high acquisition speeds also facilitates the collection of $3 \mathrm{D}$ data. As a result data collection in 3D is far more achievable on a greater range of samples.

In this contribution we will explain the analytic 3D FIB-SEM tomography workflow in detail and highlight most recent developments. Application examples with special focus on the accuracy and stability of the workflow will be presented. [2] 


\section{References:}

[1] S. Zaefferer and S. Wright in "EBSD in Material Science", ed. A. Schwartz, M. Kumar, B Adams, D. Field (Springer) p.109.

[2] Parts of the presented results were achieved in collaboration with the University of Plymouth. This collaborative work has been supported by the European Regional Development Fund.

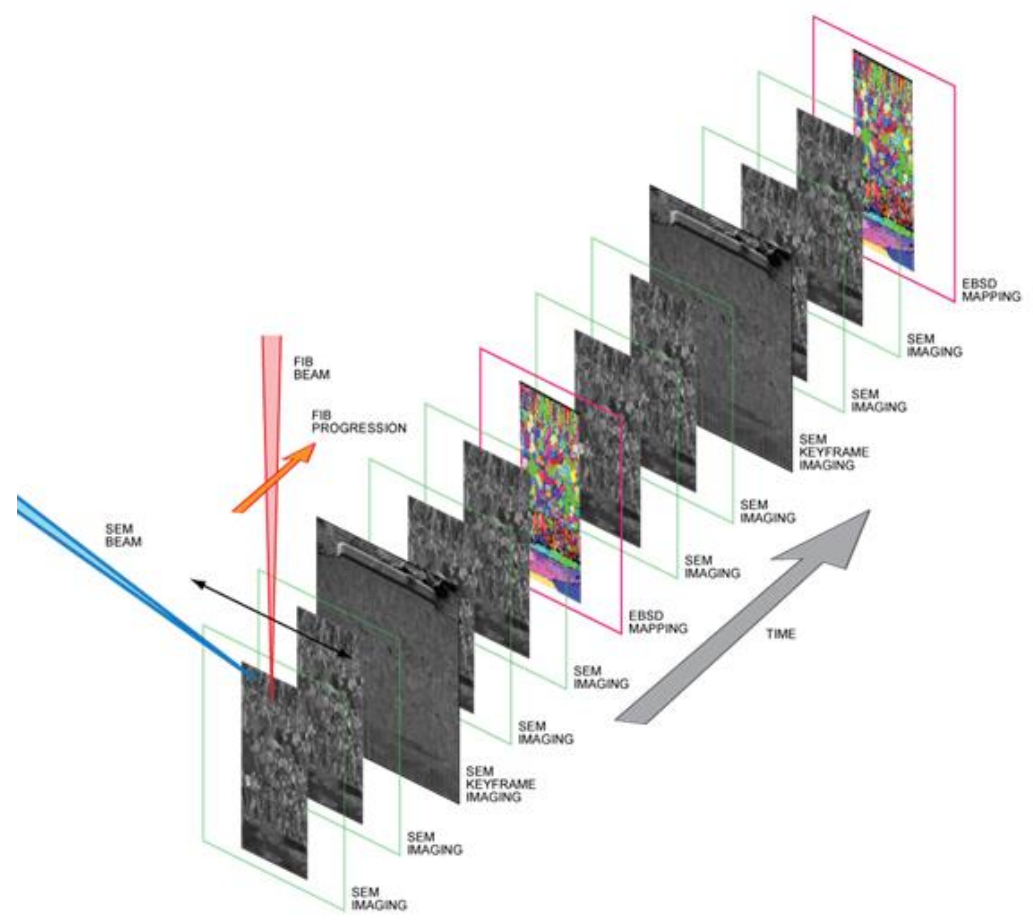

Figure 1. A schematic to illustrate the $3 \mathrm{D}$ acquisition process. The electron beam is shown in blue and the ion beam in red.

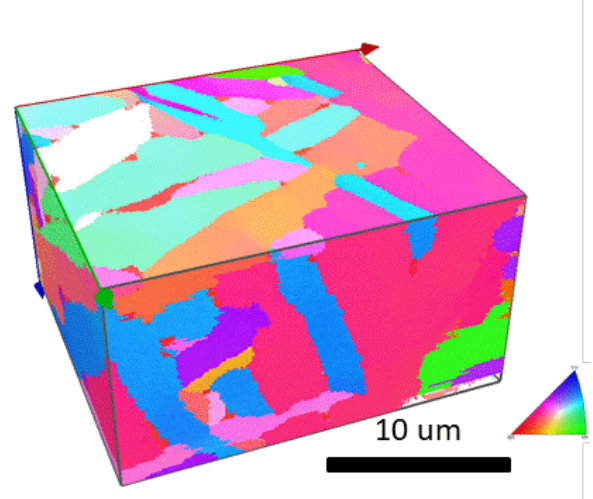

Figure 2. 3D EBSD analysis of a Ti64 alloy, data sown is an orientation map (IPF Y) and is used to study retained beta grains and grain boundaries. 